\title{
ENSURING QUALITY OF LIFE OF CANCER PATIENTS THROUGH OPTIMAL ORAL HEALTH
}

It is all about quality of life!

All clinicians are of the view that the focus of oncologist is to strive for disease-free survival of cancer patients and also unanimously agree that the maintenance of the quality of life of these patients is fundamental to our quest. Agreeing is not enough, and we must realise that an individual's quality of life is wedged from the very commencement of the oncology intervention, during which the person comes across many unexpected, lifechanging happenings. By the same token, it is, therefore, important to preserve the patients' normal functions and overall well-being.

Over the last couple of decades, there has been a steady accumulation of evidence, which indicates that oral health has a substantial influence on the quality of life. ${ }^{[1-3]}$ Good oral health allows individuals to converse effectually, to eat normally and to smile most importantly. ${ }^{[4]}$

All types of cancer and all kinds of treatments i.e., surgery, chemotherapy, radiation therapy and/or haematopoietic stem cell transplantation (HSCT) lead to consequences other than the direct and intended outcomes. Of all the problems, oral health-related side effects are the most debilitating and unfortunately the most neglected by both the patients and the healthcare providers. As oral cavity is a gateway to rest of the body, any compromise of the region leads to compromised nutrition, increase risk of oral infection, its related systemic diseases and overall decrease immune response, thus leading to long-term physical, psychological and social problems as well as delays in cure of the primary disease. ${ }^{[5]}$ The frequency of oral side effects is almost $100 \%$ in all patients of head and neck cancer, in more than $75 \%$ of patients receiving transplants and approximately $40 \%$ of the patients receiving chemotherapy with variable risk between treatment regimens. The aggressiveness of the therapy

\footnotetext{
Correspondence: Dr Ayyaz Ali Khan PhD,

Federal Postgraduate Medical Institute, Shaikh Zayed Medical

Complex, Lahore, Pakistan.

Email: ayyazalikhan@iadsr.edu.pk
}

and the quantity of radiation dose are proportionally related to the degree of side effects on dental and oral health. General side effects of cancer therapy include oral mucositis $(\mathrm{OM})$, xerostomia (salivary gland dysfunction), pain, infections, dental caries, gingival and periodontal disease, taste alterations, functional disabilities and abnormal dental development in children. Chemotherapy specifically results in neurotoxicity and gingival bleeding, while specific harmful effects of radiation include radiation-induced dental caries, trismus due to fibrosis and osteoradionecrosis of jaw bone. ${ }^{[6]}$

The good news is that most of these complications can be prevented and effectively managed before, during and after cancer treatment by following four key principles: Proper oral assessment, personalised treatment plan, proper preventive measures and adequate corrective oral healthcare. All these should be done by oral physicians as part of oncology care teams. However, in the present-day healthcare system in Pakistan, it is feasible to have the first three principles to be carried out by all healthcare providers, especially the oncology team, while the fourth objective achieved through timely patient referral to the dental professionals.

Oral assessment for cancer patients focuses on the condition of the teeth, status of the gingiva and periodontium (structures supporting the teeth), jaw bones, oral mucosa, salivary function, range of motion and presence of any prosthesis. All these parameters need to be evaluated clinically and radiographically. If no dentist is available, oncology team should do the preliminary oral evaluation. Oral hygiene instructions need to be given to all cancer patients even with a visibly disease-free oral cavity as cancer progression and its therapy will cause problems. These instructions should include the use of a soft bristle toothbrush to avoid any mucosal injury, daily floss use in areas of healthy mucosa and gingiva, daily rinse with alcohol-free mouthwashes, use of saliva substitutes in case of xerostomia and use of high fluoride toothpaste with only spitting after brushing and no rinsing with water. 
If, in preliminary assessment, patient is found to have dental caries, mobile teeth, gingival bleeding, any signs of trauma or infection, oral ulcers, mucosal redness including the dorsal surface of the tongue, difficulty in opening of mouth, dry mouth and any pain and/or loss of sensation, he/she should be referred to a dental professional for corrective action before initiating cancer treatment. All the corrective treatments are scheduled so that there is a gap of at least 2 weeks before the start of therapy allowing adequate oral healing to occur.

The corrective action by oral healthcare providers will include restoration of all teeth with cavities, removal of mobile teeth, deep scaling, root planning and curettage for the removal of dental plaque, thus reducing gingival inflammation and bleeding, treating periodontium for any infective process and prescribing exercises for improvement of mouth opening, adjustment of any illfitting dentures, instructions for proper brushing and additional use of cleaning aids and as per individual requirements of patients. In case the patient has to undergo radiation therapy for cancer of the head and neck region or spine and/or thoracic region a simple mouth guard is adequate to reduce the direct radiation effects on teeth.

Once the cancer treatment is initiated, there are more chances of OM, xerostomia, dental caries, gingival bleeding and its related side effects, and therefore, it is mandatory that patient's oral health should be monitored and recorded on each visit. Corrective action should be immediately taken during radiotherapy to treat infections and ulceration, dental caries and reduction in mouth opening in consultation with the dental team as these would compromise nutritional intake and immunity of the patients, thus delaying treatment response. In case of chemotherapy, if fever is detected and is non-traceable to any intervention, oral infection should be considered as a possible source. Vomiting during chemotherapy can cause enamel erosion and sensitivity, so patients should be advised to brush teeth after rinsing mouth with baking soda or salt. When referring for surgical dental intervention during chemotherapy, especially, in case of myelosuppressive drugs, a concerned dentist should be informed of the haematological status of the patients so that dental intervention can be catered appropriately and antibiotic prophylaxis was given if required.
$\mathrm{OM}$ is one of the most debilitating complications during cancer therapies. It can affect virtually $100 \%$ of patients receiving high-dose chemotherapy along with HSCT. This can range from mild redness and discomfort to severe ulceration. OM is graded from 1-4 (Grade 1 - soreness +/- erythema and no ulceration, Grade 2 - erythema and ulcers. Patients can swallow solid diet, Grade 3 - ulcers and extensive erythema, patients cannot swallow solid diet, and Grade 4 - alimentation [ability to take fluids and solids orally] is not possible). ${ }^{[7]}$ Grade 1 and 2 OMs can be managed by maintaining good oral hygiene, smoking cessation and mouthwashes and gels. Cocktails containing analgesics, steroids, antiallergic, antibiotic, antifungal and anaesthetic can be given to reduce symptoms and improve patient's dietary intake. In case of severe OM (Grades 3 and 4), the whole of the alimentary tract is affected and patients need to be managed with a high dose of analgesics, laxatives are given to decrease constipation and coating protectant through oral dose can be given as well. However, research is still needed to develop regimens effective against treating OM in cancer patients.

Patients reporting for follow-up should be advised to undergo dental evaluation every 4-8 weeks at least for the initial 6 months. Most of the oral side effects resolve after the chemotherapy or radiation therapy is over except in case of direct radiation to head and neck, spinal or thoracic region. Long-term effects of radiation in the region include xerostomia, osteoradionecrosis and radiation caries. The best and most cost-effective way to avoid radiation caries is to get the patient to wear a mouth guard before receiving radiation dose. For xerostomia, sipping water often and using salivary substitutes are the treatment of choice to reduce further damage caused due to dry teeth and mucosa surfaces. In patients with HCST, immunosuppression takes almost a year to recover, so they may be advised accordingly to take care of their oral hygiene and take corrective measures as and when required.

Amongst the non-communicable diseases, one of the leading causes of morbidity and mortality in Pakistan is Cancer. According to GLOBOCAN 2012, 174 new cases of cancers are diagnosed each day and approximately 133 people die daily of the disease. ${ }^{[8]}$ This burden is not expected to decrease due to aging of the population and worsening of the lifestyle factors implicated in cancer initiation and progression. Effective early interventions 
have reduced the mortality of the disease, but morbidity is still a major problem before, during and after cancer treatment all around the globe and, especially, in developing countries due to economic problems and lack of focus on multidisciplinary care.

For the healthcare providers to prevent delays in cancer treatment plans and maximise the safety and comfort of the cancer patients, it is mandatory to carry out a proper assessment and early detection of the oral health problems by the oncology team followed by personalised corrective treatment actions by oral healthcare providers. Multiple assessment tools are available for this purpose and can be filled by a trained oncology nurse. For ready reference, one can use the time tested oral assessment guide. ${ }^{[1]}$

By incorporating effective oral care to the overall treatment plan of cancer patients, we can make sure that patient will successfully complete planned cancer treatment by reducing oral pain, preventing oral infections, thus reducing serious systemic illnesses, reducing the risk of bone necrosis, optimising nutritional intake and decreasing the cost of care, thus improving the overall quality of life of our patients.

Saima Chaudhry ${ }^{1}$, Ayyaz Ali Khan ${ }^{2}$ ${ }^{1}$ Department of Oral Pathology, University of Health Sciences, Lahore, Pakistan, ${ }^{2}$ Department of Dentistry,
Federal Postgraduate Medical Institute, Shaikh Zayed Medical Complex, Lahore, Pakistan

Received: 12 December 2017 / Accepted: 21 December 2017

\section{References}

1. Eilers J, Berger AM, Petersen MC. Development, testing, and application of the oral assessment guide. Oncol Nurs Forum 1988;15:325-30.

2. Petersen PE, Bourgeois D, Ogawa $\mathrm{H}$, et al. The global burden of oral diseases and risks to oral health. Bull World Health Organ 2005;83:661-9.

3. Sheiham A. Oral health, general health and quality of life. Bull World Health Organ 2005;83:644.

4. Watt RG. Strategies and approaches in oral disease prevention and health promotion. Bull World Health Organ 2005;83:711-8.

5. Oral and Dental Care Management in Head and Neck Cancer. Clinical Practice Guideline Ver. 1. Available from: https://www. albertahealthservices.ca/assets/info/hp/ca ncer/if-hp-cancerguide-hn001-dental-oral.pdf. [Last accessed on 2017 Dec 01].

6. Cancer Treatment and Oral Health. National Instituteof Dental and Craniofacial Research. Available from: https:// www.nidcr.nih.gov/OralHealth/Topics/CancerT reatment. [Last accessed on 2017 Dec 14].

7. Mouth Care Guidance and Support in Cancer and Palliative Care. $2^{\text {nd }}$ ed. 2015. Available from: http://www.ukomic. co.uk/pdf/UK_OM_Guidelines_v 3.pdf. [Last accessed on 2017 Dec 01].

8. Sarwar MR, Saqib A. Cancer prevalence, incidence and mortality rates in Pakistan in 2012. Cogent Med 2017;4:1288773. 